\title{
Bioanalysis
}

\section{Design and statistical analysis of method transfer studies for biotechnology products}

\author{
"We present our proposals for test materials and study designs of \\ method transfer studies for biotechnology products, an equivalence \\ test for comparing means obtained in two laboratories and a \\ head-to-head approach for comparing precisions of the data obtained \\ in two laboratories."
}

First draft submitted: 30 January 2017; Accepted for publication: 21 February 2017;

Published online: 17 May 2017

Keywords: analytical method $\bullet$ equivalence testing $\bullet$ method transfer $\bullet$ statistical analysis

During biotechnology product development, the analytical instrumentation and methodology are often carefully selected based on the intended purpose and the scope of the analytical method. Specifically, parameters such as specificity, linearity, limit of detection (LOD) and limit of qualification (LOQ), range, accuracy and precision are evaluated [1]. After an analytical method is successfully validated and implemented, the updates of the method with the standard operating procedure will be conducted during the life-cycle of the product. The life-cycle management of analytical methods includes, but is not limited to, the following:

- trend analyses of the method performance at regular intervals;

- evaluation of the need to optimize the analytical method by requalification or even revalidation of all or a part of the analytical procedure due to any proposed changes (e.g., critical raw material supplier change);

- development and validation of a new or an alternative analytical method for a new impurity;
- transferring a validated analytical method from a sending laboratory to a testing laboratory.

A method transfer is common practice during the life-cycle management of pharmaceutical products. Since the analytical method to be transferred has been already thoroughly evaluated and fully validated for its intended purpose at the sending laboratory, the main purpose of method transfer studies is usually the qualification to evaluate if the two laboratories generate comparable results across the parameter ranges of interest, and to assure that the method after the transfer is still suitable for its intended use.

While the US FDA guidance to industry [1,2] and further publications [3-15] discuss the general principles for the design, analysis and evaluation of method transfer studies, they do not explicitly specify the acceptance criteria by which assay transfers are considered acceptable. Although these general principles apply to the majority of assay transfer studies, depending on the type and the intended purpose of the assays, different statistical analysis methods have been proposed to suit specific needs. Below, we briefly summarize published proposals with regard to testing materials, study
Meiyu Shen ${ }^{* 1}$ \& Lixin (Leo)

$\mathrm{Xu}^{2}$

'Division of Biometrics VI, Office of Biostatistics, Office of Translational Sciences, Center for Drug Evaluation \& Research, US FDA, 10903 New Hampshire Avenue, Silver Spring, MD 20993, USA

2Division of Drug Quality I, Office of Manufacturing Quality, Office of Compliance, Center for Drug Evaluation \& Research, US FDA, $10903 \mathrm{New}$ Hampshire Avenue, Silver Spring, MD 20993, USA

*Author for correspondence: meiyu.shen@fda.hhs.gov 
designs, parameters to be considered and statistical analysis methods.

With regard to test materials, in addition to quality control (QC) samples, samples from dosed subjects (incurred samples) are recommended [6,7]. Wieling [6] proposed to split the sample into two subsets. Lin et al. [7] recommended that bioanalytical methods use the incurred samples or a set of QC samples prepared to cover the low, medium and high concentrations in both laboratories. Samples of commercial production batches are recommended to be used [5,8-13] as testing materials. Samples from at least one batch are recommended to be tested $[4,9-12]$.

"For a well-developed and fully validated analytical method at the sending laboratory, a method transfer study should generate data to verify if the receiving laboratory can perform the analytical ", task as being executed in the sending laboratory."

With regard to the study designs, method transfer studies should evaluate the impact of factors including analysts, days, number of runs, equipment (brand and/or model), environment and reagent suppliers on the results obtained in the sending and receiving laboratories, using the same testing materials. Wieling proposed to keep the methods, chemicals, reagents, disposables, glassware, equipment (brand and model) and data reduction as identical as possible [6]. Schwenke and O'Connor proposed to evaluate the equivalence of the mean values of the results obtained from two laboratories for one analyst and then evaluate the consistency among analysts [13]. On the other hand, Altan and Shoung proposed to evaluate the equivalence of the results from two laboratories where analysts, apparatuses, batches and suppliers of analytical reagents are covariates [14].

Data from both laboratories are treated as from two independent populations in general. Several publications emphasize that the data should be paired for each of the multiple batches to evaluate the equivalence of the results between two laboratories, for example [11,12], in which case, many batches are needed.

With regard to precision, some proposed hypothesis testing while others proposed point estimate. With regard to mean values, most published studies proposed the comparison of the mean difference between two laboratories, while others proposed the comparison of the ratio of the means between two laboratories [9].

With regard to statistical analysis methods, although it has been commonly accepted that the significance test should be avoided for the comparative data in method transfer studies, several statistical analysis approaches, including the equivalence test, tolerance interval method and total error method $[3,6,8-11,13]$ have been proposed. However, these proposals did not discuss on how to establish the acceptance criteria.

In method transfer studies, some of the test system components such as analysts, analytical instrument, software/data reduction and assay controls [16] may be different while reference standard and critical reagents are the same. In each laboratory, at least two analysts should analyze a few samples from one batch with three replicates over at least 2 days. Method performance consistency should be checked with at least one more batch. Due to the sample size limit and the lack of unified approach for determining the equivalence margin, we propose a sample size-dependent margin based on the variability of the results obtained in the sending laboratory. If there are changes for a subset of test system components, the factors which do not change can be treated as constants. The proposed methods can still be applied under this condition.

We present our proposals for test materials and study designs of method transfer studies for biotechnology products, an equivalence test for comparing means obtained in two laboratories and a head-to-head approach for comparing precisions of the data obtained in two laboratories. Last, we discuss the limitations of our proposed methods and the necessity of verifying the potential bias in the transferred method in the receiving laboratory with the reference standard.

\section{Test materials \& study designs for the method transfer studies for biotechnology products}

The analytical methods of interest are intended to be used for release and stability studies of biotechnology products. Therefore, measurements from each laboratory should be obtained for one batch with multiple runs by multiple analysts and be repeated at least on another day. We may assume that the drug content within a batch is uniform as the drug content uniformity within a batch for any biotechnology products should not be a challenge since they are typically developed using a liquid formulation and the uniformity should be confirmed (or verified) by the manufacturers before releasing any intended products. Even with lyophilized formulation (powder), when being reconstituted, the solution will be checked for a series of quality attributes including content uniformity. For each batch, the measurements at two laboratories are considered independent when the same analytical method is implemented at two laboratories and different analysts perform analytical tasks using the same or different instruments and reagents. Forced degradation samples or samples of a drug substance or a drug product containing pertinent product-related impurities should be used if the transferred assay is a stability-indicating method. 


\section{Equivalence test for comparing means obtained in two laboratories}

For a well-developed and fully validated analytical method at the sending laboratory, a method transfer study should generate data to verify if the receiving laboratory can perform the analytical task as being executed in the sending laboratory. One way to accomplish this purpose is to compare the means obtained in two laboratories using an equivalence test with appropriate equivalence margins. Mathematically, the equivalence test has been formulated with the following hypotheses. Denote the means of the response variable of interest by $\mu_{R}$ and $\mu_{S}$, respectively, for the receiving laboratory and the sending laboratory.

$$
\begin{aligned}
& \mathrm{H}_{0}: \mu_{\mathrm{R}}-\mu_{\mathrm{S}} \leq-\delta \text { or } \mu_{\mathrm{R}}-\mu_{\mathrm{S}} \geq \delta \\
& \mathrm{H}_{\mathrm{a}}:-\delta<\mu_{\mathrm{R}}-\mu_{\mathrm{S}}<\delta
\end{aligned}
$$

Here $\delta$ is a prespecified constant, also called an equivalence margin.

The null hypothesis, $\mathrm{H}_{0}$, states that $\mu_{\mathrm{R}}$ and $\mu_{\mathrm{S}}$ are not equivalent. The alternative hypothesis for equivalence, $\mathrm{H}_{\mathrm{a}}$, is the intersection of the two one-sided parameter regions, $\left\{-\delta<\mu_{\mathrm{R}}-\mu_{\mathrm{s}}\right\}$ and $\left\{\mu_{\mathrm{R}}-\mu_{\mathrm{s}}<\delta\right\}$.

An appropriate value for $\delta$ for different analytical methods is hard to determine and a unified approach of obtaining a value for $\delta$ for different analytical methods has not been proposed yet. For example, the equivalence margin developed from HPLC may not be suitable for a bioassay transfer study or vice versa, since the data from bioassay methods are often more variable and those obtained by HPLC method is less variable. The determination of an appropriate value for $\delta$ is critical, since too small value may require unrealistic number of observations for passing the equivalence test and too large value may falsely assure good performance of the transferred method at the receiving laboratory, and that could potentially result in releasing poor quality products to the given target or population.

In practice, the analysts would verify the performance of the transferred method at the receiving laboratory using 20-30 observations per laboratory. Based on this consideration, we propose $\delta$ as $C \sigma_{S}$, for example $\mathrm{C}=0.5-1, \sigma_{\mathrm{S}}$ is the standard deviation obtained in the original laboratory. No expert in biological sciences can accept $\delta>\sigma_{S}$ used for the method transfer studies, since the method transfer is acceptable when the method performance is equal or better in the receiving laboratory for the purpose of product QC. Long-term QC data may not be appropriate for determining $\sigma_{S}$, since the QC may include too many unknown factors, that is, if there is a product stability trend over time or if there is a data drift from the time of the assay implementation. It is reasonable to use the data from side-by-side comparative method transfer studies for estimating $\sigma_{s}$. Note that all equivalence margins should be smaller than the ranges specified in product release specification.

In the following subsections, we will discuss statistical analyses for the equivalence test with a known value for $\delta$ and then discuss how to select an appropriate margin for the equivalence test based on the practical sample sizes.

\section{Statistical analyses for the equivalence test with a known value for $\delta$}

Let $\overline{\mathrm{X}}_{\mathrm{s}}$ and $\hat{\sigma}_{\mathrm{s}}^{2}$ be respectively the sample mean and sample variance of $n_{S}$ independent and identical observations. These observations obtained at the sending laboratory follow the normal distribution with mean $\mu_{\mathrm{s}}$ and vari-

ance $\sigma_{\mathrm{S}}^{2}$, denoted as $\mathrm{N}\left(\mu_{\mathrm{S}}, \sigma_{\mathrm{S}}^{2}\right)$. Let $\overline{\mathrm{X}}_{\mathrm{R}}$ and $\hat{\sigma}_{\mathrm{R}}^{2}$ be respectively the sample mean and sample variance of $n_{R}$ obser-

vations from $N\left(\mu_{R}, \sigma_{R}^{2}\right)$ obtained at the receiving laboratory, where mean $\mu_{\mathrm{R}}$ is the population mean and $\sigma_{\mathrm{R}}^{2}$ is the population variance of measurements obtained at the receiving lab. Then we can define test statistics

values $T_{1}=\frac{\bar{X}_{R}-\bar{X}_{s}-\delta}{\sqrt{\frac{\hat{\sigma}_{R}^{2}}{n_{R}}+\frac{\hat{\sigma}_{S}^{2}}{n_{s}}}}$ and $\quad T_{2}=\frac{\bar{X}_{R}-\bar{X}_{s}-\delta}{\sqrt{\frac{\hat{\sigma}_{R}^{2}}{n_{R}}+\frac{\hat{\sigma}_{S}^{2}}{n_{s}}}}$ as the basis for hypothesis test (Equation 1). $T_{1}$ and $T_{2}$ follow approximate $t$-distribution with $\mathrm{v}$ degrees of freedom, where $\mathrm{v}$ is approximated by Satterthwaite approximation [17]. We reject $\mathrm{H}_{0}$ in (Eeuation 1) if $\mathrm{T}_{1}>\mathrm{t}_{\alpha}(\mathrm{v})$ and $\mathrm{T}_{2}<-\mathrm{t}_{\alpha}(\mathrm{v})$, where $t_{\alpha}^{0}(v)$ is the 1- $\alpha$ quantile of t-distribution with degrees of freedom $v, \alpha$ is the nominal significance level (e.g., 0.05).

We define the power, ${ }^{\mathrm{P}}\left(\theta^{*}, \sigma, \mathrm{n}, \delta, \alpha\right)$, as the probability to reject $\mathrm{H}_{0}$ in (EQuation 1) and conclude that the means obtained in two laboratories are equivalent. To simplify the calculation of sample sizes using $\mathrm{P}\left(\theta^{*}, \sigma, n, \delta, \alpha\right)$, we assume that $\sigma_{\mathrm{R}}=\sigma_{\mathrm{S}}=\sigma$ and $\mathrm{n}_{\mathrm{R}}=\mathrm{n}_{\mathrm{S}}=\mathrm{n} . \quad \mathrm{P}\left(\theta^{*}, \sigma, \mathrm{n}, \delta, \alpha\right) \quad$ is expressed in (EQuation 2) when the true difference in the means obtained in two laboratories is $\theta^{*}$.

$\mathrm{P}\left(\theta^{*}, \sigma, n, \delta, \alpha\right)$

$=\int_{0}^{c_{0}}\left(\Phi\left(\frac{\delta-\theta^{*}}{2 \sigma \sqrt{1 / n}}-t_{\alpha}(v) \sqrt{\frac{x}{v}}\right)-\Phi\left(\frac{-\delta-\theta^{*}}{2 \sigma \sqrt{1 / n}}+t_{\alpha}(v) \sqrt{\frac{x}{v}}\right)\right) \frac{1}{2^{v / 2} \Gamma(v / 2)} x^{v / 2-1-1} e^{-x / 2} d x$

where $\Phi($.$) is the normal cumulative function,$ 


$$
\mathrm{c}_{0}=\delta^{2} \mathrm{vn} /\left(4 \mathrm{t}_{\alpha}^{2}(\mathrm{v}) \sigma^{2}\right) \text { and } \mathrm{v}=\mathrm{n}-2
$$

Statistical analyses for the equivalence test with $\mathrm{C}_{\mathrm{s}}$ for $\delta$

Below we determine the value for $\mathrm{C}$, which is used to calculate the equivalence margin $\delta=C \sigma_{S}$, considering the sample size. When $\theta^{*}=0, \quad \sigma_{\mathrm{R}}=\sigma_{\mathrm{S}}=\sigma$ and $\mathrm{n}_{\mathrm{R}}=\mathrm{n}_{\mathrm{S}}=\mathrm{n}$, the power function

becomes

$\mathrm{P}\left(\theta^{*}=0, \sigma, \mathrm{n}, \delta=\mathrm{C} \sigma, \alpha\right)$

$=\int_{0}^{c_{0}}\left(\Phi\left(\frac{C}{2 \sqrt{1 / n}}-t_{\alpha}(v) \sqrt{\frac{x}{v}}\right)-\Phi\left(\frac{-C}{2 \sqrt{1 / n}}+t_{\alpha}(v) \sqrt{\frac{x}{v}}\right)\right) \frac{1}{2^{v / 2} \Gamma(v / 2)} x^{v / 2-1} e^{-x / 2} d x$

where $\mathrm{c}_{0}=\mathrm{C}^{2} \mathrm{vn} /\left(4 \mathrm{t}_{\alpha}^{2}(\mathrm{v})\right)$ and $\mathrm{v}=\mathrm{n}-2$.

The $\mathrm{C}$ value changes from 0.99 to 0.80 as $n$ increases from 20 to 30 to achieve $85 \%$ power when $\theta^{*}=0$. Hence, $\mathrm{C}=0.85$ is reasonably chosen such that we can achieve about $85 \%$ power with a sample size in the range 20-30 per laboratory.

One analysis is carried out as the margin $\mathrm{C}_{\mathrm{s}}$ is the known value, although $\sigma_{S}$ is estimated with the data obtained in the sending laboratory in the method transfer study. Since this analysis inflates both the probability of rejecting $\mathrm{H}_{0}$ under $\mathrm{H}_{a}$ (Type I error rate) and the probability of failing to reject $\mathrm{H}_{0}$ under $\mathrm{H}_{a}$ (type II error rate), we recommend that the margin be estimated with the data and Wald method [18] be used for hypothesis test in (EQuation 1). Then we can define test statistics values $\mathrm{T}_{1}^{\prime}=\frac{\overline{\mathrm{X}}_{\mathrm{R}}-\overline{\mathrm{X}}_{\mathrm{S}}+\mathrm{C} \hat{\sigma}_{\mathrm{S}}}{\sqrt{\hat{\sigma}_{\mathrm{R}}^{2} / \mathrm{n}_{\mathrm{R}}+\hat{\sigma}_{\mathrm{S}}^{2} / \mathrm{n}_{\mathrm{S}}+\mathrm{C}^{2} \hat{\sigma}_{\mathrm{S}}^{2}\left(\mathrm{C}_{0}-1\right)}}$ and $\mathrm{T}_{2}^{\prime}=\frac{\overline{\mathrm{X}}_{\mathrm{R}}-\overline{\mathrm{X}}_{\mathrm{S}}+\mathrm{C} \hat{\sigma}_{\mathrm{S}}}{\sqrt{\hat{\sigma}_{\mathrm{R}}^{2} / \mathrm{n}_{\mathrm{R}}+\hat{\sigma}_{\mathrm{S}}^{2} / \mathrm{n}_{\mathrm{S}}+\mathrm{C}^{2} \hat{\sigma}_{\mathrm{S}}^{2}\left(\mathrm{C}_{0}-1\right)}}$, where $\mathrm{C}_{0}=\sqrt{\frac{\mathrm{n}_{\mathrm{s}}-1}{2}} \Gamma\left(\frac{\mathrm{n}_{\mathrm{s}}-1}{2}\right) / \Gamma\left(\frac{\mathrm{n}_{\mathrm{s}}}{2}\right)$. We reject $\mathrm{H}_{0}$ in (Equation 1) if $\mathrm{T}_{1}^{\prime}>\mathrm{Z}_{\alpha}$ and $\mathrm{T}_{2}^{\prime}<-\mathrm{Z}_{\alpha}$.

\section{Head-to-head approach for comparing precisions obtained in two laboratories}

Since the equivalence test focuses on the mean differences between two laboratories, we should also conduct head-to-head analysis comparing precisions of the data from the two laboratories. We may set up the hypothesis test for comparing precisions of the data from the two laboratories as:

$\mathrm{H}_{0}: \sigma_{\mathrm{R}} \leq \sigma_{\mathrm{S}}$

$\mathrm{H}_{\mathrm{a}}: \sigma_{\mathrm{R}}>\sigma_{\mathrm{S}}$

The power to reject $\mathrm{H}_{0}$ in (Eguation 4) may be smaller than the power to reject $\mathrm{H}_{0}$ in (EQUation 1) since the vari- ability of a variance estimate may be much larger than the variability of a mean estimate. In order to reject $\mathrm{H}_{0}$ in (EQuation 4), we need to increase sample sizes used for hypothesis test (EQuation 1). Without increasing sample sizes, the hypothesis testing for (EеUation 4) would result in concluding that the precision of the data from the receiving laboratory is better than that from the sending laboratory due to insufficient power to reject the null hypothesis.

We may conduct the equivalence test for comparing the precision of the data from two laboratories, but then we must face the challenge of selecting an appropriate margin and finding the correct test statistics.

Considering all challenges discussed above, we recommend comparing the sample estimate of the variability of the data from the receiving laboratory $\left(\hat{\sigma}_{\mathrm{R}}\right)$ with the sample estimate of the variability of the data from the sending laboratory $\left(\hat{\sigma}_{\mathrm{S}}\right)$. We conclude that the precision obtained from the receiving laboratory is at least as good as that obtained from the sending laboratory if $\hat{\sigma}_{\mathrm{R}} \leq \hat{\sigma}_{\mathrm{S}}$.

\section{Discussion}

Our proposal of equivalence testing with the equivalence margin as a function of practical sample sizes fits for the use in the practice; others' proposal of equivalence testing does not have unified equivalence margin or does not provide methods to determine the criteria. For example, Dewe et al. [9] proposed the total error approach with a $\beta$-expectation tolerance interval method, in which a proportion $(\beta)$ of the new laboratory population is expected to fall within the acceptance limits around the mean estimate of the sending laboratory $\left([1-\gamma] \mu_{s},[1+\gamma] \mu_{s}\right)$ where $\mu_{s}$ is the mean of the sending laboratory, and a risk assessment approach, in which the method transfer is accepted if the probability of having results outside the acceptance limits is less than $1-\beta$. The performance of this approach depends on the acceptance limits $\left([1-\gamma] \mu_{s},[1+\gamma] \mu_{s}\right)$ and prespecified $\beta$. However, no discussion is provided on how to determine $\beta$ and $\gamma$.

Frömke et al. [11] proposed splitting one sample into two parts (one analyzed by the sending laboratory and the other analyzed by the receiving laboratory) and conducting the equivalence test on the ratio of means obtained in two laboratories, and performing a noninferiority test on the ratio of the variances obtained in two laboratories. No discussion is provided on the equivalence margin and the noninferiority margin. Again the performance of these methods depends on the equivalence margin and noninferiority margin.

Zhong et al. [12] proposed the tolerance interval approach to assure more than $100 \mathrm{P} \%$ of the individual difference (or percent difference, d) between individual results obtained in the sending laboratory 
and the receiving laboratory are within the predefined boundary (L, U) with $100(1-\alpha) \%$ confidence level. Here $0<\alpha<1,0<\mathrm{P}<1$ and $\mathrm{P}$ is the proportion of the population within (L, U). In their example, one pair of observations for each of 36 batches was obtained by two laboratories. No discussion was provided about how to determine $\mathrm{L}$ and $\mathrm{U}$, as well as $\mathrm{P}$. The performance of tolerance interval depends on $\mathrm{L}$, $\mathrm{U}$ and $\mathrm{P}$.

Agut et al. [15] discussed a panel of historical strategies once used by their company, for example, the interlaboratory studies with more than one receiving laboratories. They further proposed an equivalence test on the mean differences between the sending and receiving laboratories. The $90 \%$ upper confidence limit for the coffient of variation $(\mathrm{CV})$ of the intermediate precision of the receiving laboratory was compared with the $\mathrm{CV}$ criterion. The equivalence acceptance criterion for the mean and the criterion for $\mathrm{CV}$ were determined from high probability of passing a good batch with respect to the release specification limits and high probability of failing a bad batch with respect to the release specifications. Their way of determining the equivalence margin and CV criterion leaves no variability from processes, incoming materials and others. The performance of their equivalence testing depends on the value of their high probability and their definition of good and bad products.

Okamoto [19] proposed a fixed acceptance criterion for a specific assay in the evaluation of the method transfer of HPLC. However, these fixed values for HPLC are not applicable to any bioassay since the bioassay has more variability than HPLC does.

From above discussion, we propose the equivalence test for hypothesis (Equation 1) with a margin $\left(\delta=C \hat{\sigma}_{\mathrm{S}}\right.$, $C<1$, e.g., $C=0.85$ ) estimated from the method transfer studies to compare the means of the data obtained from two defined laboratories in method transfer stud- ies, in which practical sample sizes are in the range of 20-30 for one batch per laboratory. One disadvantage of the proposed method is that $C \hat{\sigma}_{\mathrm{S}}$ can be larger than the specification. In this case, $\delta$ should be reduced to a value smaller than the specification. We recommend Wald test for this equivalence test since the sample size is reasonably large. We also propose a head-tohead comparison of the precision of the data from the two laboratories. Method performance consistency should be checked with at least one more batch. We must point out that we should check the accuracy using a known reference standard to determine if the mean of data from the receiving laboratory meets the accuracy criteria.

\section{Acknowledgements}

The authors would like to thank $Y$ Tsong (Office of Biostatistics, Office of Translational Sciences, Center for Drug Evaluation and Research, US FDA), J Liu and C Torigoe (Office of Biotechnology Products, Center for Drug Evaluation and Research, FDA), for their scientific insight through many discussions, comments and edits. The authors would like to thank the anonymous reviewers and commissioning editor for their comments and help.

\section{Disclaimer}

This article reflects the views of the authors and should not be construed to be those of the US FDA.

\section{Financial \& competing interests disclosure}

The authors have no relevant affiliations or financial involvement with any organization or entity with a financial interest in or financial conflict with the subject matter or materials discussed in the manuscript. This includes employment, consultancies, honoraria, stock ownership or options, expert testimony, grants or patents received or pending, or royalties.

No writing assistance was utilized in the production of this manuscript.

\section{References}

1 Draft Guidance on Analytical Procedures and Methods Validation for Drugs and Biologics. US Food and Drug Administration, USA (2014).

www.pharmalytik.com/images/stories/Guidance

2 International Society for Pharmaceutical Engineering. The Good Practice Guide: Technology Transfer International society for pharmaceutical engineering, FL, USA (2003).

3 United States Pharmacopeia. Transfer of analytical procedure. 37-National Formulary 32.

https://hmc.usp.org/sites/default/files/documents/HMC

4 Ermer J, Limberger M, Lis K, Wätzig H. The transfer of analytical procedures. J. Pharm. Biomed. Anal. 85, 262-276 (2013).
5 Briggs J, Nicholson R, Vazvaei F et al. Method transfer, partial validation, and cross validation: recommendations for best practices and harmonization from the global bioanalysis consortium harmonization team. AAPS J. 16(6), 1143-1148 (2014).

6 Wieling J. Robust, fit-for-purpose method transfer: why we should apply equivalence testing. Bioanalysis 7(7), 807-814 (2015).

7 Lin Z, Li W, Weng N. Capsule review on bioanalytical method transfer: opportunities and challenges for chromatographic methods. Bioanalysis 3(1), 57-66 (2011).

8 Chambers D, Kelly G, Limentani G, Lister A, Lung KR, Warner E. Analytical Method Equivalency: An Acceptable Analytical Practice [PhD Thesis]. Pharmaceutical Technology, NJ, USA (2005). http://images.alfresco.advanstar.com/alfresco_images 
9 Dewé W, Govaerts B, Boulanger B, Rozet E, Chiap P, Hubert P. Using total error as decision criterion in analytical method transfer. Chemometrics and Intelligent Laboratory Systems 85(2), 262-268 (2007).

10 Kaminski L, Schepers U, Wätzig H. Analytical method transfer using equivalence tests with reasonable acceptance criteria and appropriate effort: extension of the ISPE concept. J. Pharm. Biomed. Anal. 53(5), 1124-1129 (2010).

11 Frömke C, Hothorn LA, Sczesny F, Onken J, Schneider M. Analytical method transfer: improving interpretability with ratio-based statistical approaches. J. Pharm. Biomed. Anal. 74, 186-193 (2013).

12 Zhong JL, Lee K, Tsong Y. Statistical assessment of analytical method transfer. J. Biopharm. Stat. 18(5), 1005-1012 (2008).

13 Schwenke JR, O'Connor DK. Design and analysis of analytical method transfer studies. J. Biopharm. Stat. 18(5), 1013-1033 (2008).
14 Altan S, Shoung JM. Block designs in method transfer experiments. J. Biopharm. Stat. 18(5), 996-1004 (2008).

15 Agut C, Caron A, Giordano C, Hoffman D, Ségalini A. Transfer of analytical procedures: a panel of strategies selected for risk management, with emphasis on an integrated equivalence-based comparative testing approach. J. Pharm. Biomed. Anal. 56(2), 293-303 (2011).

16 Krause S. Validation of Analytical Methods for Biopharmaceuticals: A Guide to Risk-based Validation and Implementation Strategies. PDA/DHI, IL, USA (2007).

17 Satterthwaite FE. An approximate distribution of estimates of variance components. Biometrics 2(6), 110-114 (1946).

18 Chen Y, Weng Y, Dong X, Tsong Y. Wald tests for varianceadjusted equivalence assessment with normal endpoints. J. Biopharm. Stat. 27 (2),308- 316 (2017).

19 Okamoto M. Assay validation and technology transfer: problems and solutions. J. Pharm. Biomed. Anal. 87, 308-312 (2014). 